\title{
Effects of shadowing in oblique-incidence metal (100) epitaxial growth
}

\author{
Yunsic Shinf and Jacques G. Amal \\ Department of Physics \& Astronomy \\ University of Toledo, Toledo, $\mathrm{OH} 43606$
}

(Dated: September 15, 2018)

\begin{abstract}
The effects of shadowing in oblique incidence metal (100) epitaxial growth are studied using a simplified model. We find that many of the features observed in $\mathrm{Cu}(100)$ growth, including the existence of a transition from anisotropic mounds to ripples perpendicular to the beam, can be explained purely by geometrical effects. We also show that the formation of (111) facets is crucial to the development of ripples at large angles of incidence. A second transition to 'rods' with (111) facets oriented parallel to the beam is also found at high deposition angles and film thicknesses.
\end{abstract}

PACS numbers: 81.15.Aa, 68.55.-a, 81.10.Aj

While a variety of surface relaxation processes, such as adatom diffusion on terraces and near steps, as well as edge-diffusion and detachment, are usually assumed to determine the surface morphology in epitaxial growth, recently it has been shown ${ }^{1.2,3.4}$ that the deposition process can also play an important role. For example, in the case of unstable metal epitaxial growth with an Ehrlich-Schwoebel (ES) barrier ${ }^{5}$ to diffusion over descending steps, the short-range (SR) attraction of depositing atoms to the substrate can significantly enhance the surface roughness and selected mound angle. $\underline{\underline{4}}$ In addition, for sufficiently large angles of incidence, the deposition angle can also play an important role. For example, in recent experiments on $\mathrm{Cu} / \mathrm{Cu}(100)$ epitaxial growth 1.2 at $250 \mathrm{~K}$ a gradual transition was observed from symmetric mound structures for deposition angles up to $\theta=55^{\circ}$ (where $\theta$ is the angle between the beam and the substrate normal), to asymmetric mounds with increasing slopes for deposition angles up to $70^{\circ}$, to asymmetric ripples oriented perpendicular to the beam with $(113) /(111)$ facets on the shadow/illuminated sides at $\theta=80^{\circ}$. Similar results have been obtained in grazing incidence $\mathrm{Co} / \mathrm{Cu}(001)$ growth $^{6}$ for which it was found that the resulting surface anisotropy also leads to strong uniaxial magnetic anisotropy. Thus, understanding the effects of oblique incidence deposition is important since it may lead to the possibility of controlling both the surface morphology and magnetic properties in epitaxial growth.

Although the effects of shadowing on thin-film morphology have been extensively studied in the case of amorphous and polycrystalline columnar growth, $\underset{7}{\underline{7}}$ the case of epitaxial growth is not as well understood. We note that in Ref. 2 it was shown that at large angles of incidence the long-range (LR) van der Waals attraction of depositing atoms to the substrate may play an important role in addition to the SR attraction. Therefore, a fully realistic simulation of oblique-incidence epitaxial growth can be very time-consuming since it must take into account both the LR and SR interactions. As a result, recent simulations have focused on the submonolayer regime $e^{8}$ or at most on the first few layers of vicinal growth $\frac{9}{9}$ However, at high angles of incidence the effects of shadowing and crystal geometry also play an increas- ingly important role. Therefore, it is of interest to determine to what extent these purely geometric effects may determine the surface morphology.

Here we present the results of simulations carried out using a simplified model of fcc(100) epitaxial growth in which the effects of shadowing are included but not the additional modifying effects of the SR and LR attraction. Our results indicate that many of the qualitative and semi-quantitative features observed in $\mathrm{Cu}(100)$ growth, $, 1,2$ including the existence of anisotropy in the submonolayer regime, can be explained purely by geometrical (shadowing) effects. In addition, we find that the formation of (111) facets is crucial to the formation of ripple structures at large angles of incidence. The dependence of the critical thickness for ripple formation on deposition angle is also studied and good scaling behavior is obtained for the correlation length perpendicular to the beam as a function of deposition angle and film thickness. At higher thicknesses we also observe a second transition to 'rods' with (111) facets oriented parallel to the beam. We also find that the surface width increases exponentially with deposition angle and exhibits excellent scaling as a function of film thickness.

Except for deposition, our model is very similar to previous models 10.11 used to study metal (100) growth at normal incidence in which the correct crystal geometry has been taken into account. In particular, atoms are deposited with a (per site) deposition rate $F$, while adatoms (monomers) on a flat terrace are assumed to diffuse with hopping rate $D$. Since the ES barrier typically plays an important role in metal epitaxial growth, the rate for an adatom at a descending step-edge to diffuse over the step is given by $D_{E S}=D e^{-E_{E S} / k_{B} T}$ where $E_{E S}$ is the Ehrlich-Schwoebel barrier. Compact islands are also assumed and accordingly a moderate amount of edge- and corner-diffusion is also included, while the attachment of atoms to existing islands is assumed to be irreversible. Thus the most important parameters in our model are the deposition angle $\theta$, the ratio $D / F$ of the monomer diffusion rate to the deposition rate, and the magnitude of the ES barrier.

To take into account the effects of shadowing and crystal geometry, each deposited atom is assumed to travel 

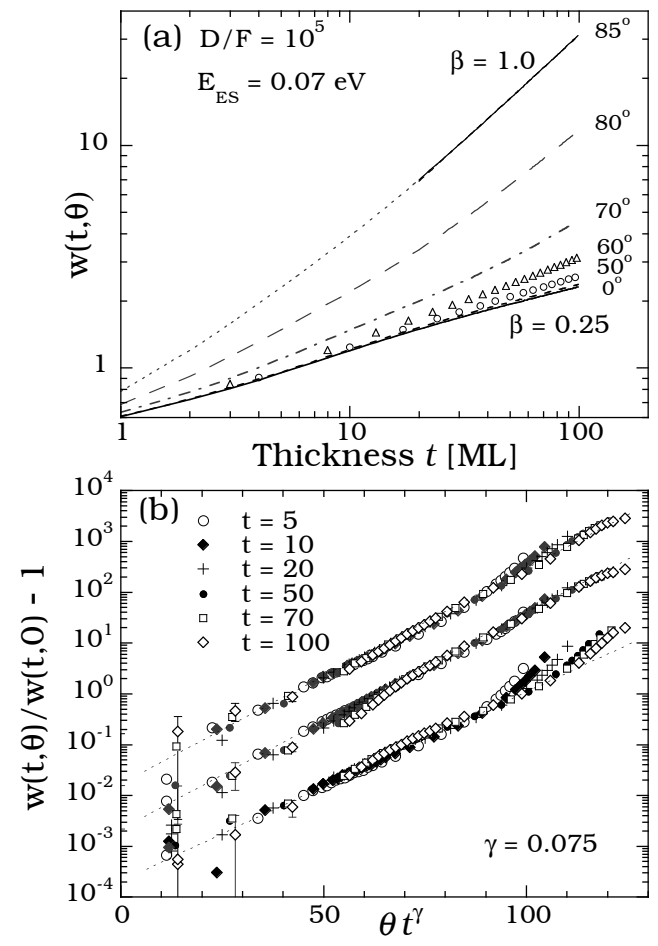

FIG. 1: (a) Surface roughness (in ML) as function of film thickness for $\theta=0^{\circ}-85^{\circ}$ for case of slow deposition and moderate ES barrier (b) Relative width deviation $\delta w / w(t, 0)$ as function of scaled deposition angle $\theta t^{\gamma}$ for $\theta=10^{\circ}-88^{\circ}$ and $t=5-100 \mathrm{ML}$ for three different cases: fast deposition, high ES barrier (top), fast deposition moderate ES barrier (middle), and slow deposition, moderate ES barrier (bottom).

ballistically until its distance to the closest substrate atom is less than or equal to the nearest-neighbor distance. The depositing atom then 'cascades' randomly via downward funneling $(\mathrm{DF})^{12}$ from a site corresponding to this atom until it reaches a four-fold hollow site. Thus, in our model atoms deposited on (111) facets are assumed to diffuse essentially instantaneously to the terrace below. Given the extremely low barriers for diffusion on metal (111) surfaces (approximately $0.05 \mathrm{eV}$ for $\left.\mathrm{Cu}(111)^{13}\right)$ for temperatures which are not too low this is a very reasonable approximation.

In most of our simulations two different deposition rates were used - one corresponding to a 'slow' deposition rate $\left(D / F=10^{5}\right)$ and the other corresponding to a 'fast' deposition rate $(D / F=5000)$. Similarly, two different values of the ES barrier were also used, one corresponding to a 'moderate' barrier at room temperature $\left(E_{E S}=0.07 \mathrm{eV}\right)$ and the other corresponding to a large, effectively infinite ES barrier. As in the experiments of van Dijken et al $\mathrm{l}^{1.2}$ the overall deposition rate was assumed to be independent of deposition angle. Similarly, the azimuthal angle was chosen such that the deposition direction was parallel to the close-packed step-edge, i.e. along the [110] direction.

In order to understand the dependence of the surface morphology on deposition conditions we have calculated a variety of different quantities as a function of average film thickness $t$ (where $t$ is in ML) and deposition angle $\theta$. These include the r.m.s. surface height or "width" $w$, the lateral correlation lengths $\xi_{\|}$and $\xi_{\perp}$ determined from the zero-crossing of the height-height correlation functions parallel and perpendicular to the beam, and the anisotropy $\alpha=\xi_{\perp} / \xi_{\|}$. Our simulations were typically carried out using relatively large system sizes $(L=512)$ and averaged over 100 runs.

Fig. 1(a) shows typical results for the surface width as a function of film thickness for deposition angles ranging from $0^{\circ}$ (normal incidence) to $85^{\circ}$, for the case of slow deposition and a moderate ES barrier. As in the $\mathrm{Cu}(100)$ growth experiments of van Dijken et al, $\stackrel{1}{,}$ for $\theta \leq 50^{\circ}$ the effects of oblique incidence and shadowing on the surface roughness are relatively weak. However, for larger deposition angles the surface roughness increases significantly with increasing deposition angle. As a result, the value of the effective roughening exponent $\beta$ at large film thickness $t$ (where $w \sim t^{\beta}$ ) increases from $\beta \simeq 1 / 4$ for small angles to a value close to 1 at $\theta=85^{\circ}$.

Fig. 1(b) shows the corresponding results for the relative deviation in the width $w(t, \theta)$ compared to the width at normal incidence as a function of the scaling variable $\theta t^{\gamma}$, where the scaling exponent $\gamma \simeq 0.075$. Also included are similar results obtained for the cases of fast deposition with a moderate ES barrier, and fast deposition with a large ES barrier, which have been shifted up by factors of 10 and 100 respectively for clarity. As can be seen, there is excellent scaling for all three sets of deposition conditions. In addition, we note that the (unshifted) scaling functions in all three cases are almost identical except for some small deviations for large $\theta t^{\gamma}$. The linearity of the scaling function in each case over approximately 4 decades shows that for fixed deposition rate the relative width deviation increases exponentially with increasing deposition angle $\theta$. The small value of the scaling exponent $\gamma$ also indicates that the surface width depends much more strongly on deposition angle than on film thickness.

Fig. 2 shows typical pictures of the surface morphology at a film thickness of 50 ML for different deposition angles for the case of slow deposition (moderate ES barrier). At a deposition angle $\theta \simeq 70^{\circ}$ (Fig. 2(a)) shadowing leads to a preference for mounds to coalesce along the direction perpendicular to the beam and the onset of ripple formation. At a somewhat larger angle $\left(\theta \simeq 80^{\circ}\right)$ asymmetric ripples with (111) facets on the illuminated side and (113) facets on the shadow side are formed (see Fig. 2(b) and inset) as in the experiments of Dijken et al $\stackrel{1.2}{2}$ The asymmetry of these ripples can be more clearly seen at $\theta \simeq 85^{\circ}$ (Fig. 2(c)) which shows clearly the (111) facets on the illuminated side. We note that at this angle there is also evidence for a competition between the growth of asymmetric ripples perpendicular to the beam direction, and the growth of 'rods' with (111) facets parallel to the beam. In simulations with reduced corner 


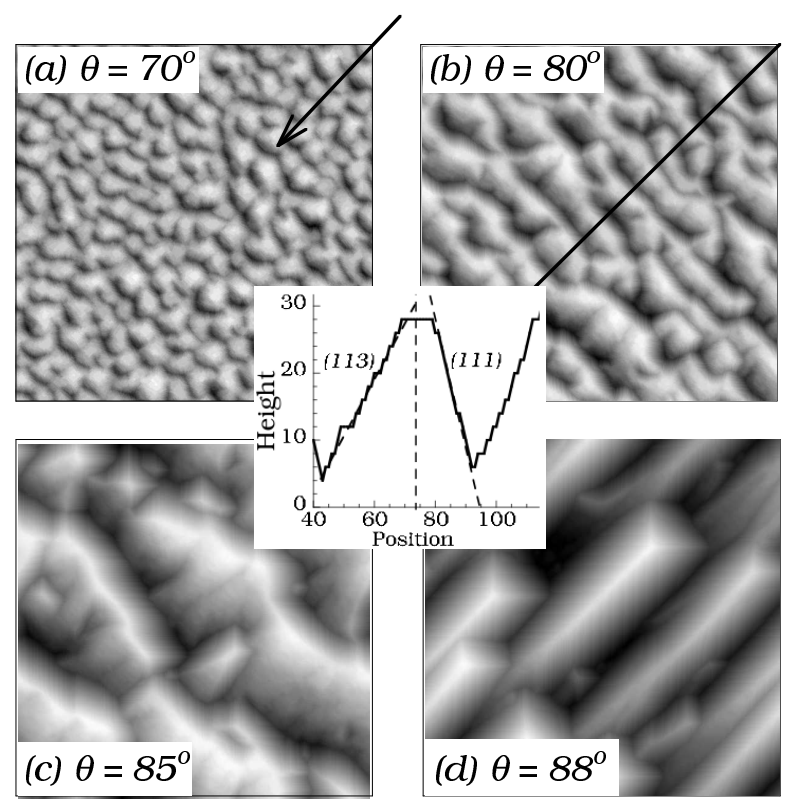

FIG. 2: Gray-scale pictures of surface morphology (system size $L=512$ ) for same conditions as in Fig. 1(a) at $t=50$ ML. Arrow indicates deposition direction. Inset shows ripple profile taken along line in (b).

diffusion this competition leads to an extended regime in which isotropic pyramidal structures with (111) facets on all four sides are formed, similar to what is observed experimentally in Ref. 1. Finally, at $\theta=88^{\circ}$ (Fig. 2(d)) the perpendicular ripples are completely replaced by the growth of 'rods' with (111) facets growing parallel to the beam.

The dependence of the anisotropy on both deposition angle and film thickness for this set of deposition conditions can be seen more quantitatively in Fig. 3. At a critical thickness $t_{c}$, which increases rapidly with decreasing deposition angle, there is an abrupt increase in the anisotropy, corresponding to the onset of ripple formation. The anisotropy then saturates as the ripples continue to elongate as well as coarsen but then decreases as $t^{-1 / 2}$ at higher film thicknesses. As can be seen in Fig. 3(b), at large angles there is already a small but noticeable anisotropy in the submonolayer regime, even in the absence of steering effects due to SR and LR attraction ${ }^{14}$ This anisotropy is primarily due to the fact that shadowing tends to inhibit (enhance) the coalescence of islands along the directions parallel (perpendicular) to the beam.

A detailed examination of the surface morphology in the multilayer regime indicates that the abrupt increase of the anisotropy and formation of ripples is due to the formation of (111) facets on the illuminated sides of mounds. Since (111) facets can efficiently capture and transport depositing atoms to the sides, this leads to a strong enhancement of mound coalescence in the direction perpendicular to the beam, followed by the forma-
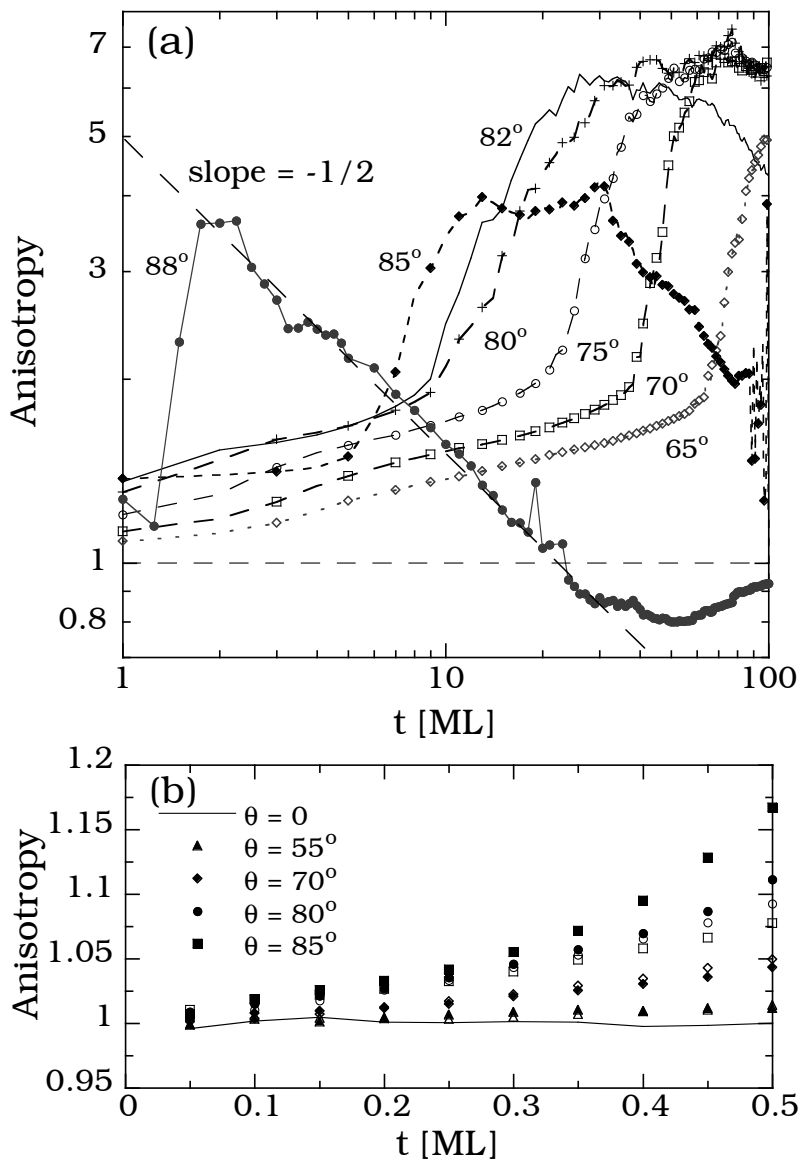

FIG. 3: Anisotropy $\alpha=\xi_{\perp} / \xi_{\|}$as a function of film thickness for the case of slow deposition with a moderate ES barrier. (a) multilayer regime (b) submonolayer regime. In (b) the corresponding results for a large ES barrier are also shown (open symbols).

tion and growth of ripples with extended (111) facets on the illuminated side. However, the saturation and eventual decrease of the anisotropy may be attributed to the formation of structures with (111) facets on the sides parallel to the beam as well as on the illuminated side (see Fig. 2(d)) whose growth competes with ripple growth. Such structures are particularly stable at large deposition angle, while due to the large flux on the illuminated facet they tend to grow linearly in the direction of the beam. The decay of the anisotropy with exponent $-1 / 2$ (see Fig. 3(a)) may then be explained by the fact that in this regime the correlation length perpendicular to the beam grows as $\xi_{\perp} \sim t^{1 / 2}$ while the correlation length parallel to the beam grows linearly with film thickness, e.g. $\xi_{\|} \sim t$.

Fig. 4 shows the scaled perpendicular correlation length as a function of scaled deposition angle $\theta t^{2 \gamma}$ where $\gamma \simeq 0.075$, for $t=5-100 \mathrm{ML}$ and $\theta=55^{\circ}-85^{\circ}$ for three different sets of deposition parameters (see caption) where (a) and (b) have been shifted upwards by factors of 100 and 10 for clarity. As can be seen, in each case there is excellent scaling over a wide range of film 


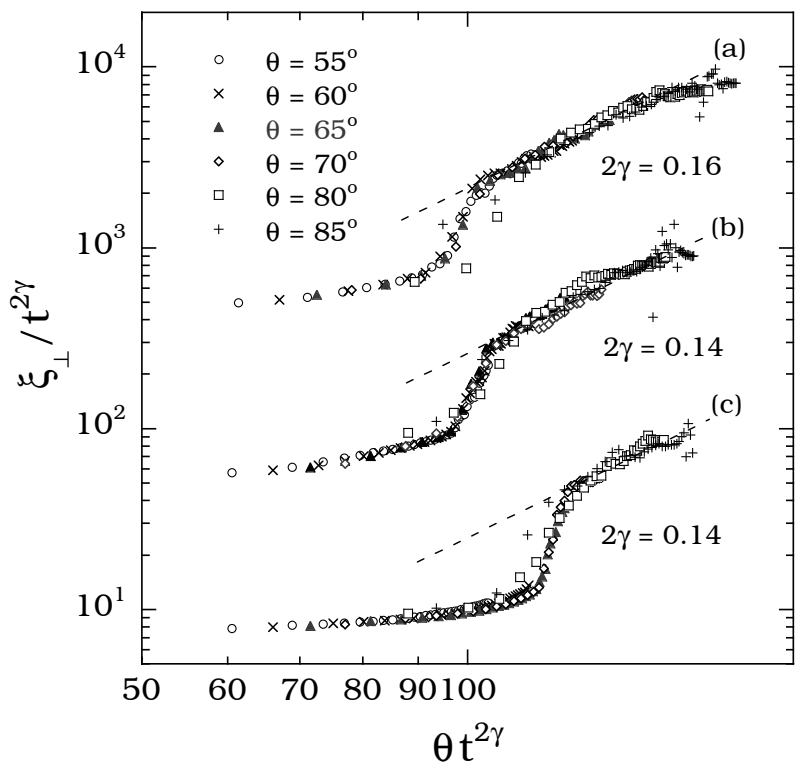

FIG. 4: Scaled correlation length $\xi_{\perp} / t^{2 \gamma}$ as function of scaled deposition angle for three different sets of deposition parameters: (a) fast deposition, large ES barrier (b) fast deposition, moderate ES barrier, and (c) slow deposition, moderate ES barrier. Slope of dashed lines is approximately 2.6.

thicknesses and deposition angles. This scaling behavior also indicates that ripple formation may be expected to occur for deposition angles as low as $55^{\circ}$, although the relatively low value of $\gamma$ indicates that the critical thickness for ripple formation increases rapidly with decreasing deposition angle. We note that the slope of the scaling function after the onset of ripple formation (dashed lines in Fig. 4) corresponds to the $t^{1 / 2}$ growth of ripples with (111) facets perpendicular to the beam, and is consistent with domain growth with non-conserved order parameter, 15 while the later 'saturation' corresponds to the slower coarsening of structures with (111) facets parallel to the beam, and is consistent with one-dimensional fluctuation induced coarsening $\underline{\underline{16}}$ We also find excellent scaling for the scaled correlation length $\xi_{\perp} / l_{d}$ as a function of the scaled film thickness $t / l_{d}^{2}$ where $l_{d} \simeq(D / F)^{1 / 6}$ is the diffusion length, although the resulting scaling function depends on deposition angle.

Finally, we discuss the factors that may affect ripple formation in oblique-incidence epitaxial growth. Since ripple formation is associated with the formation of (111) facets on the illuminated sides of mounds, we expect that anything that promotes this tends to enhance ripple formation. For example, since a large ES barrier leads to faster mound formation as well as a larger selected mound slope, increasing the ES barrier leads to earlier ripple formation as shown in Fig. 4. Similarly, decreasing the flux and thus increasing $D / F$, or increasing the rates of edge- and/or corner-diffusion delays the formation of (111) facets and ripples as again shown in Fig. 4. We have also considered the case of low-temperature growth $(D / F \simeq 0)$ but assuming that $\mathrm{DF}$ as well as fast diffusion on (111) facets remain active. Somewhat surprisingly, we find that even in this case, for which the surface is 'sta-

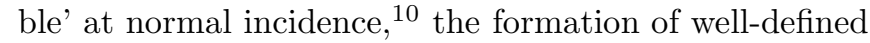
ripples with (111) facets is observed at large deposition angles $\left(\theta \geq 60^{\circ}\right)$. Thus, we conclude that while ripple formation may be enhanced by the presence of a mound instability, it is primarily a geometric effect due to shadowing as well as the existence of rapid diffusion on (111) facets.

In conclusion, we have used a simplified model to study the evolution of the surface morphology and anisotropy in oblique-incidence metal (100) growth. Our results indicate that much of the qualitative and even semiquantitative behavior can be explained by geometric effects which dominate at large deposition angles. Our results also indicate the existence of a competition between ripples perpendicular to the beam and structures with (111) facets parallel to the beam which eventually leads to a novel transition from perpendicular ripples to 'rods' at high deposition angles and film thicknesses. While we expect that the inclusion of SR and LR attraction will affect the results presented here, the qualitative picture is unlikely to change. In particular, due to the extreme sensitivity on deposition angle, the most significant effects of including such interactions are likely to be a slight shift in the angles at which ripple and/or rod formation take place as well as a slight modification of the anisotropy. It is also possible that the inclusion of the SR and LR attraction may further stabilize the "isotropic phase" corresponding to the competition between rods and ripples as is observed experimentally. In future work, we plan to carry out multiscale simulations in order to determine in more detail to what extent the effects of SR and LR attraction may modify this picture.

\section{Acknowledgments}

This research was supported by grants from the Petroleum Research Fund and NSF as well as by a grant of computer time from the Ohio Supercomputer Center.
* Electronic address: yshim@physics.utoledo.edu

$\dagger$ Electronic address: jamar@physics.utoledo.edu

1 S. van Dijken, L.C. Jorritsma, and B. Poelsema, Phys. Rev. Lett. 82, 4038 (1999).
2 S. van Dijken, L.C. Jorritsma, and B. Poelsema, Phys. Rev. B 61, 14047 (2000).

${ }^{3}$ F. Montalenti and A.F. Voter, Phys. Rev. B 64, 081401 (R) (2001). 
4 J. Yu and J. G. Amar, Phys. Rev. Lett. 89, 286103 (2002).

5 G. Ehrlich and F. G. Hudda, J. Chem. Phys. 44, 1039 (1966); R. L. Schwoebel, J. Appl. Phys. 40, 614 (1969).

6 S. van Dijken, G. Di Santo, and B. Poelsema, Appl. Phys. Lett. 77, 2030 (2000).

7 See for example, D-X Ye, Y-P Zhao, G-R Yang, Y-G Zhao, G-C Wang, and T-M Lu, Nanotechnology 13, 615 (2002) and references therein.

8 J. Seo, S.-M. Kwon, H.-Y. Kim, and J.-S. Kim, 67, 121402 (R) (2003).

9 J. Seo, H.-Y. Kim, and J.-S. Kim, Phys. Rev. B 71, 075414 (2005).

10 J. G. Amar and F. Family, Phys. Rev. B 54, 14742 (1996).

11 Y. Shim and J.G. Amar, Phys. Rev. B 73, 035423 (2006).
12 J. W. Evans, D. E. Sanders, P. A. Thiel, and A. E. DePristo, Phys. Rev. B 41, R5410 (1990).

13 A. Bogicevic, S. Ovesson, P. Hyldgaard, B.I. Lundqvist, H. Brune, and D.R. Jennison, Phys. Rev. Lett. 85, 1910 (2000).

14 In the submonolayer regime the anisotropy was estimated by calculating the ratio of the average island lengths perpendicular and parallel to the beam respectively.

15 A.J. Bray and A.D. Rutenberg, Phys. Rev. E 49 R27 (1994).

16 L.-H. Tang, P. Smilauer, and D.D. Vvedensky, Eur. Phys. J. B 2409 (1998). 